\title{
microRNA-1 inhibits cardiomyocyte proliferation in mouse neonatal hearts by repressing CCND1 expression
}

\author{
Jingyi Gan ${ }^{1,2 \#}$, Florence Mei Kuen Tang ${ }^{1 \#}$, Xianwei Su${ }^{3}$, Gang Lu ${ }^{3}$, Jing Xu ${ }^{2}$, Henry Siu Sum Lee \\ Kenneth Ka Ho Lee ${ }^{1,5}$
}

${ }^{1}$ MOE Key Laboratory for Regenerative Medicine, School of Biomedical Sciences, The Chinese University of Hong Kong, Hong Kong, China; ${ }^{2}$ Department of Basic Medicine, School of Medicine, Xi'an International University, Xi'an 710077, China; ${ }^{3}$ CUHK-SDU Joint Laboratory on Reproductive Genetics, School of Biomedical Sciences, The Chinese University of Hong Kong, Hong Kong, China; ${ }^{4}$ Botnar Research Centre, NIHR Oxford Biomedical Research Unit, University of Oxford, Oxford, UK; ${ }^{5}$ Chinese University of Hong Kong-University of Southampton Joint Laboratory for Regenerative Medicine, School of Biomedical Sciences, The Chinese University of Hong Kong, Hong Kong, China

Contributions: (I) Conception and design: KK Lee, J Gan; (II) Administrative support: KK Lee; (III) Provision of study materials or patients: KK Lee, G Lu; (IV) Collection and assembly of data: J Gan, FM Tang, X Su, HS Lee; (V) Data analysis and interpretation: All authors; (VI) Manuscript writing: All authors; (VII) Final approval of manuscript: All authors.

"These authors contributed equally to this work.

Correspondence to: Kenneth Ka Ho Lee. School of Biomedical Sciences, Chinese University of Hong Kong, Room 127, 1/F, Lo Kwee-Seong Integrated Biomedical Sciences Building, Hong Kong, China. Email: kaholee@cuhk.edu.hk.

Background: The functions of microRNA-1 (miR-1) in cardiac hypertrophy, and cardiomyocyte differentiation have been investigated. However, the mechanism on how miR-1 could repress cardiomyocyte proliferation has not been fully elucidated.

Methods: We address this issue by investigating whether miR-1 affected the proliferation of neonatal cardiomyocyte and identify some of the genes targeted by miR-1. miR-1 was over-expressed in neonatal cardiomyocytes and the effect on cell cycle and growth were analyzed by flow cytometry and Brduincorporation assay. Relevant vectors carrying the luciferase reporter were constructed for validation of miR-1 binding to its matching sites on the 3'-untranslated region of the predicated target mRNAs. Cardiomyocytes were co-transfected with the vectors and miR-1 mimics, then luciferase reporter assay was performed. Lastly, we examined the expression of target genes in cardiomyocytes after transfection with miR-1 mimics, as well as their normal expression pattern in 2- and 13-day-old mice hearts.

Results: We have demonstrated that miR-1 was the most significantly upregulated miRNA in 13-day-old mouse hearts compared with 2-day-old hearts. We also showed that miR-1 could repress cardiomyocyte G1/S phase transition, proliferation and viability. IGF1 and CCND1 were identified as candidate target genes regulated by miR-1. In addition, overexpression of miR-1 could suppress the expression of these two genes at the mRNA level. It could also correspondingly inhibit CCND1 expression at the protein level but not for IGF1.

Conclusions: Our results suggest that miR-1 plays an important role in inhibiting cardiomyocyte proliferation in the developing neonatal mouse heart by directly suppressing the cell-cycle regulator, CCND1.

Keywords: microRNA-1 (miR-1); cardiomyocyte proliferation; CCND1; neonatal mouse heart

Submitted Jan 06, 2019. Accepted for publication Jun 17, 2019.

doi: $10.21037 /$ atm.2019.08.68

View this article at: http://dx.doi.org/10.21037/atm.2019.08.68 


\section{Introduction}

Previous studies have shown that urodele amphibians and zebrafish have extraordinary abilities to regenerate their heart following injuries $(1,2)$. In adult zebrafish, they can regenerate their heart after $20 \%$ of the ventricle was removed. This was achieved without fibrotic scar formation and mainly through cardiomyocyte proliferation. However, this remarkable ability is not seen in mammalian hearts. It has been reported that cardiomyocytes in 2 day-old mouse neonates could still divide extensively in all regions of the heart $(3,4)$. Unfortunately, this proliferative ability is greatly reduced by the time that the neonatal heart reaches 13 days old. In the adult mammalian heart, the cardiomyocytes are "irreversibly" growth arrested so are unable to selfrenewal following myocardial damage. Surprisingly, Porrello et al. (5) reported that 1-day-old mouse neonates could completely regenerate their myocardium after $15 \%$ of their left ventricle was removed. Detailed analyses of the results revealed that the regeneration was achieved through the proliferation of pre-existing cardiomyocytes, while the architecture of the heart and physiological functions were also restored. However, 7-day-old neonates lose this regenerative ability and fibrotic scars replace the missing myocardium. In this context, it is important to elucidate the underlying mechanisms that regulate neonatal cardiomyocyte proliferation, which could potentially be translated in regenerative medicine to treat degenerative heart diseases.

Eulalio et al. (6) have reported that two miRNAs (hsamiR-590 and hsa-miR-199a) possess the ability to facilitate adult cardiomyocytes' re-entry into the cell cycle ex vivo. In addition, following myocardial infarction in mice, expression of these miRNAs could be used as a marker to assess the extent of myocardial recovery. It has been reported that miR-25 could potently delay calcium influx in cardiomyocytes in vitro and increased the risk of heart failure in humans and mice (7). Furthermore, injection of antagomiR against miR-25 could prevent heart failure in mice by improving cardiac function and cardiomyocyte survival. It now appears that altered patterns of miRNAs expression are not only involved in a variety of cardiac disorders (8) but also demonstrated promising results in enhancing cardiac regeneration.

miRs belong to a class of single strand nucleotides, which are about $22 \mathrm{nt}$ long and are composed of highly conserved small non-coding RNAs that regulate gene expression at post-transcriptional level (9). Mature miRNAs function by binding to the 3'-untranslated region of target genes that contain the complementary base-paring. The pairing process results in transcriptional repression or degradation of the target mRNAs, which may be involved in organ development, cell proliferation, differentiation and apoptosis-mainly through the negative regulation of protein expression (10). miRs' sequences are highly conservative in different species, demonstrating tissuespecific and developmental stage-specific expressions. They are adept at regulating the expression of transcription factors and signaling mediators, which play imperative roles in the cardiac development processes and proper timely functions. Presently, we investigated and identified miRNAs distinctly expressed in 2-day-old and 13-day-old mice hearts. Furthermore, we also examined the relationship between these differentially expressed miRs and their target mRNAs with respect to cardiomyocytes proliferation.

\section{Methods}

\section{Animals}

Two- and 13-day-old ICR neonates were obtained from Laboratory Animal Services Centre, Chinese University of Hong Kong. All experimental protocol used in this study was approved by the Chinese University of Hong Kong Animal Care and User Committee and Department of Health (Ref No.: 13-2 in DH/HA\&P/8/2/1 Pt.28).

\section{Primary cardiomyocyte cultures}

Primary cardiomyocyte cultures were established from 2-day-old mouse neonate hearts. Briefly, the hearts were isolated from the mice, dissected, rinsed and minced in HEPES-buffered saline solution (HBSS). The tissues were then enzymatic dissociated using $0.1 \%$ collagenase $\mathrm{I}$ in HBSS at $37{ }^{\circ} \mathrm{C}$ for $1 \mathrm{hr}$. The dissociated cell suspension was centrifuged at 1,000 rpm for $5 \mathrm{~min}$ and resuspended in DMEM/ F12, (Invitrogen) containing 10\% fetal bovine serum, 100 units $/ \mathrm{ml}$ penicillin and $100 \mathrm{mg} / \mathrm{mL}$ streptomycin. Subsequently, the cells were maintained in a humidified incubator at $37^{\circ} \mathrm{C}$ and $5 \% \mathrm{CO}_{2}$.

\section{Tissue cryo-sectioning and immunofluorescent staining}

Briefly, 2- and 13-day-old mice hearts were freshly harvested, fixed in $4 \%$ paraformaldehyde and dehydrated in $30 \%$ sucrose solution overnight (11). The hearts were then 
embedded in OCT and sectioned at $5 \mu \mathrm{m}$ on a Cryotome (ThermoFisher Scientific, USA). The sections were mounted onto glass slides and then washed twice in PBS for 10 minutes. For immunofluorescence staining, the sections were first permeabilized in $0.3 \%$ Triton $\mathrm{X}-100$ and $0.1 \%$ SDS in PBS solution for $30 \mathrm{~min}$, followed by blocking in $2 \%$ BSA plus $5 \%$ donkey serum for $1 \mathrm{hr}$. The sections were then stained overnight at $4{ }^{\circ} \mathrm{C}$ with the following primary antibodies (1:200) diluted in blocking solution: mouse anticardiac troponin T antibody (cTnT, Abcam), Rabbit Ki67 antibody (Abcam) and Rabbit pH3 antibody (Santa Cruz Biotechnology). The tissues were washed three times with PBST (PBS buffer with $0.05 \%$ Tween 20) for $15 \mathrm{~min}$ to remove unbound primary antibodies. Then the appropriate secondary antibody (1:300) conjugated to Alexa Fluor-488, -555 or -647 (Life Technologies) was added for $1 \mathrm{hr}$ at room temperature, in the dark with gentle shaking. The unbound secondary antibody was removed by washing with PBST, three times for $10 \mathrm{~min}$ and PBS for $5 \mathrm{~min}$. The nuclei were counter-stained with 4, 6-diamidino-2-phenylindole (DAPI, Molecular Probes) in 70\% (v/v) glycerol and mounted with glass cover slips. Immunofluorescence images were captured under a confocal microscope (FV1000; Olympus, Japan) with fixed exposure settings for all of the samples (12).

\section{miR-1 mimics and antagomir functional analysis}

miR-1 mimics and antagomir were purchased from GenePharma $^{\mathrm{TM}}$ (Shanghai, China) and used for the functional analysis. microRNA sequences were generated from Caenorbabdities elegans and served as a negative control (N.C.) in the mimics and antagomir experiments. The sequences are listed in Table S1. The cell transfection experiments were performed using Lipofectamine ${ }^{\circledR}$ RNAiMAX Reagent (Invitrogen Life Technologies, USA) and instructions according to the manufacturer. Briefly, $3.5 \times 10^{5}$ cardiomyocytes were seeded into each well of a 6-well culture plate. These cells were then transfected with miR-1 mimics, mimics N.C. (control), anti-miR-1 and antimiR N.C. (control), using Lipofectamine RNAiMAX.

\section{Stem-loop quantitative real-time miRNA assay}

Total RNA, including microRNA, were extracted from heart tissue samples or cultured cardiomyocytes using a NucleoSpin Total RNA Isolation Kit (Clontech Laboratories, USA), according to the manufacturer's instruction. After DNase I (Takara, Japan) digestion for
15 min, the RNA concentration was determined using a NanoDrop $^{\mathrm{TM}} 2000$ Spectrophotometer (ThermoFisher Scientific, USA). Realtime miRNA assay was performed in two steps: stem-loop RT reaction and quantitative realtime (qRT) PCR detection. RNA was reverse-transcribed into cDNA with specific RT primers (Table S1) by using the RevertAid First Strand cDNA Synthesis Kit (Thermo Scientific, USA). RT-qPCR was then performed to assay miRNA expression using the specific forward primers and the universal reverse primer complementary to the anchor primer. PCRs were conducted in triplicates using an ABI 7900HT Real-Time PCR Detection System (Applied Biosystems, USA) and $2 \times$ SYBR Premix Ex Taq (Takara, Japan) at $95^{\circ} \mathrm{C}$ for $30 \mathrm{~s}, 40$ cycles of $95^{\circ} \mathrm{C}$ for $5 \mathrm{~s}$ and $60{ }^{\circ} \mathrm{C}$ for $30 \mathrm{~s}$. After RT-qPCR amplification, a dissociation analysis (melt curve) was performed to identify the characteristic peaks associated with primer-dimers in order to separate them from the single prominent peak that represents the successful PCR amplification of miRs. Relative expression level of a given miRNA was determined by delta-delta $(\Delta \Delta)$ Ct calculation and reference to normalized to U6 snRNA control level. Expression levels of miRs were presented as fold change $\left(2^{-\Delta \Delta C t}\right)$. The data was expressed as mean and standard deviation of relative values obtained from three different samples. $T$-tests were performed with significant $\mathrm{p}$-value set at 0.05 for comparison. The PCR primers for mature miR1 or U6 were designed as follows: miR-1 forward primer, 5'-CGGCGGTGGAATGTAAAGAAG-3' and miR-1 reverse primer 5'-GTGCAGGGTCCGAGGT-3'. U6 snRNA forward primer, 5'-TTCCTTGGCCCCTGCGCA-3' and reverse primer, 5'-GTGCAGGGTCCG AGGT-3'. Other miRs primer sequences are reported in Table S1.

\section{RT-qPCR assays}

Total RNA was extracted from cultured cardiomyocytes and heart tissue samples as described above. One $\mu \mathrm{g}$ of total RNA was reverse-transcribed into cDNA with oligo $\left(\mathrm{dT}_{18}\right)$ primer using the RevertAid First Strand cDNA Synthesis Kit (Thermo Scientific, USA) according to the manufacturer's instruction. Briefly, $20 \mu \mathrm{L}$ of the reactant was incubated for $60 \mathrm{~min}$ at $42{ }^{\circ} \mathrm{C}, 5 \mathrm{~min}$ at $70^{\circ} \mathrm{C}$, and then stored at $-20^{\circ} \mathrm{C}$. A SYBR Green Master Mix Kit was used in the qRT-PCR to quantify expression of the target genes. GAPDH mRNA levels were used for normalization. The analysis methods used was similar to that mentioned in the Stem-loop Quantitative Realtime miRNA Assay. The 
primer sequences used analyzing murine FGF4, Gas211, IGF-1, Frs2, FoxP1 Cdc14a, CCND2, CCND1 and CDC14a expression are listed in Table S1.

\section{Cell viability assay}

MTT assays were performed to establish the viability of neonatal cardiomyocytes after miR-1 mimics and antagomir treatments. Briefly, $200 \mu \mathrm{L}$ of $1 \times 10^{4}$ neonatal cardiomyocytes was seeded into each well of a 96-well plate. These cells were allowed to attach for $24 \mathrm{hr}$ and then transfected with $100 \mathrm{nmol} / \mathrm{L}$ miR-1 mimics, mimics negative control (N.C.). After $48 \mathrm{hr}$ of transfection, $20 \mu \mathrm{L}$ of $5 \mathrm{mg} / \mathrm{mL}$ MTT (Sigma, USA) was added to the cultures and incubated for another $4 \mathrm{hr}$ at $37^{\circ} \mathrm{C}$ and $5 \% \mathrm{CO}_{2}$. The MTT solution was then removed and the precipitated formazan crystals were dissolved in $200 \mu \mathrm{L}$ DMSO. The optical density (OD) value for the dissolved formazan was measured on a microplate reader (Bio-Rad) with wavelength set at $490 \mathrm{~nm}$.

\section{BrdU cell proliferation analysis}

The effect of miR-1 on cardiomyocytes proliferation was investigated using Bromodeoxyuridine (BrdU) incorporation analysis. Briefly, cardiomyocytes were transfected with $100 \mathrm{nmol} / \mathrm{L}$ miR-1 mimics or negative mimic control and maintained in growth medium for $48 \mathrm{hr}$. The cells were then pulse labeled for $4 \mathrm{hr}$ in the presence of $10 \mu \mathrm{M}$ of $\mathrm{BrdU}$ introduced into the culture medium. After pulse labelling, the cells were fixed and processed for immunoflorescent staining using mouse monoclonal BrdU antibody (1:200, Invitrogen). The BrdU incorporation rate was determined as the percentage of $\mathrm{BrdU}^{+}$cells over the total number of DAPI+ cells in each randomly selected microscopic field. On average, 10 microscopic fields from three independent experiments were evaluated.

\section{Flow cytometry analysis}

The cell cycles of control and experimental cardiomyocytes were assessed and determined using flow cytometry. These cells were plated into 6 -well plates at $3.5 \times 10^{5}$ cells per well and allowed to grow for $24 \mathrm{hr}$. The cells were transfected with $100 \mathrm{nmol} / \mathrm{L}$ miR-1 mimics, negative mimic control (N.C.), miR-1 antagomir and antagomir N.C. for $48 \mathrm{hr}$ using Lipofectamine RNAiMAX Reagent (Invitrogen,CA). The cells were then trypsinized, collected by centrifugation, washed in cold PBS and fixed overnight at $4{ }^{\circ} \mathrm{C}$ in $70 \%$ ethanol/PBS. Subsequently, the cells were centrifuged and washed twice with PBS and stained with $20 \mu \mathrm{g} / \mathrm{mL}$ propidium iodide (Sigma-Aldrich, USA), $100 \mu \mathrm{g} / \mathrm{mL}$ RNase (Roche) and $0.1 \%$ Triton-X100 to a final volume of $200 \mu \mathrm{L}$ at room temperature for $30 \mathrm{~min}$. The cell cycle was analyzed using a flow cytometer (FACS Calibur, Becton Dickinson, USA) at $542 \mathrm{~nm}$. The experiment was conducted three times and at least 10,000 events were analyzed for each sample. The cell phase percentages were processed and determined using a Modfit software.

\section{Immunocytofluorescent staining}

Cardiomyocytes $\left(0.75 \times 10^{4}\right.$ per well $)$ were seeded onto $\phi 1.2 \mathrm{~cm}$ glass coverslips (Thermo Scientific, USA) in a 24-well tissue culture plate for $24 \mathrm{hr}$. The cells were transfected with $100 \mathrm{nmol} / \mathrm{L}$ miR-1 mimics and N.C. for $48 \mathrm{hr}$ as described above. Subsequently the samples were washed twice with PBS and fixed in 4\% paraformaldehyde for $30 \mathrm{~min}$. The cells were then permeabilized with $0.3 \%$ Triton X-100 and $0.1 \%$ SDS in PBS solution for $30 \mathrm{~min}$, followed by blocking in 2\% BSA with $5 \%$ donkey serum for $1 \mathrm{hr}$. These cells were stained overnight at $4{ }^{\circ} \mathrm{C}$ with the following primary antibodies (1:200 dilution in blocking solutions): Mouse Anti-Cardiac Troponin T antibody (Abcam), Rabbit Anti-IGF1 antibody (Abcam), Rabbit pH3 antibody (Santa Cruz Biotechnology) and Rabbit Anti-CCND1 antibody. The specimens were then washed three times with PBST (PBS buffer with $0.05 \%$ Tween 20) for 15 min to remove unbound primary antibody. Then treated with the appropriate secondary antibody (1:300 Abcam) conjugated to Alexa Fluor- $488,-555$ or -647 (Life Technologies) was added for $1 \mathrm{hr}$ at room temperature in the dark on a shaker. All unbound secondary antibodies were removed by washing with PBST (3X) for $10 \mathrm{~min}$ and PBS for $5 \mathrm{~min}$. The nuclei were counterstained with DAPI (Molecular Probes, USA) in 50\% (v/v) glycerol and mounted onto the glass slides. The stained specimens were analyzed and recorded on a confocal microscope (FV1000; Olympus, Japan).

\section{Predication of target genes regulated by miR-1}

miRNAs function by binding to the 3'UTR of their target mRNAs which causes the mRNA to degrade or represses their translation. We have searched the potential target genes of miR-1 using online softwares and databases 
(TargetScan, PicTar, miRBase and miRanda). We found that the mature sequence of miR-1 was highly conserved between different species, including mice, rat and human. Among the 695 predicted conserved targets, we highlighted FGF4, Gas211, IGF-1, Frs2, FoxP1 Cdc14a, CCND2, CCND1 and CDC14a, which playing important roles cell proliferation and differentiation.

\section{Construction of luciferase reporters}

We have constructed vectors that included the 3'UTR binding site of FGF4, Frs2, FoxP1 or CCND1 mRNAs to examine whether miR-1 could directly bind these 4 mRNAs in cardiomyocytes. The 3'UTR segments (400-600 bp) of target mRNAs, predicted to interact with miR-1, were amplified by PCR from cDNA that was reversely transcribed from RNA extracted from the 2-dayold hearts. The PCR primers used during amplification are listed in Table S1. The amplified fragments were individually subcloned upstream of the luciferase coding sequence of Firefly/Renilla dual reporter vector pmirGLO (Promega, Madison, USA). The correct insertions of vectors were confirmed by sequencing (BGI, China).

\section{Dual-glo luciferase reporter assays}

The direct interaction between miR-1 and 3'UTR of FGF4, Frs2, FoxP1 or CCND1 mRNAs were assessed. Neonatal cardiomyocytes were transiently co-transfected with $100 \mathrm{ng}$ recombinant luciferase vectors (containing IGF1, Frs2, FoxP1, CDK9 or CCND1-3'UTR) and $100 \mathrm{nM}$ miR-1 mimics or mimics N.C. using Lipofectamine 2000 (Invitrogen). At $48 \mathrm{hr}$ transfection, luciferase activity was measured using the Dual-Glo luciferase assay system (Promega, Medison). The firefly luciferase activity was normalized against renilla luciferase for each individual analysis. The values in the miR-1 mimics group were compared with those transfected with mimics N.C. and pmirGLO empty vector. The results were presented as mean \pm standard deviation (SD) from triplicated experiments.

\section{Western blot analysis}

Cardiomyocytes transfected with $100 \mathrm{nmol} / \mathrm{L}$ miR-1 mimics or negative control were washed twice with cold PBS and lysed on ice in $200 \mu \mathrm{L}$ RIPA buffer $(50 \mathrm{mM}$ sodium chloride, 1\% Nonidet P-40, $20 \mathrm{mM}$ Tris, $\mathrm{pH}$ 7.6) in the presence of protease inhibitor cocktail (Complete ULTRA
Tablets, Roche Applied Science, USA) and $100 \mu \mathrm{g} / \mathrm{mL}$ PMSF for $30 \mathrm{~min}$. The cell lysates were then centrifuged at $12,000 \mathrm{rpm}$ at $4^{\circ} \mathrm{C}$ for $20 \mathrm{~min}$. The protein supernatant was transferred into Eppendorf tubes and stored at $-80{ }^{\circ} \mathrm{C}$. The protein concentration of each sample was measured using a Bio-Rad protein assay kit (Bio-Rad, USA). Equal amount $(50 \mu \mathrm{g})$ of cell lysates was loaded onto $10 \%$ SDSPAGE and run for $2 \mathrm{hr}$ at constant voltage (stacking gel: 80V, $30 \mathrm{~min}$; separating gel: 200V, $90 \mathrm{~min}$ ). A Trans-Blot semi-dry electrophoretic transfer cell (Bio-Rad) was used to electrophoretically transfer the separated proteins onto PDVF membranes (GE Healthcare), which were then blocked with $5 \%$ skimmed milk in TBST $(10 \mathrm{mM}$ Tris $\mathrm{pH}$ 7.6, $50 \mathrm{mM} \mathrm{NaCl}, 0.05 \%$ Tween-20) for $1 \mathrm{hr}$ at room temperature. The membranes were first incubated with 1:1,000 diluted primary antibodies (anti-CCND1, anti-IGF1, anti-Tubulin $\beta 3$ ) at $4{ }^{\circ} \mathrm{C}$ overnight. The blots were then washed with TBST (TBS buffer with $0.05 \%$ Tween 20), then probed with IRDye $800 \mathrm{CW}$-labeled secondary antibodies and IRDye 680LT-labeled secondary antibodies (Li-COR, USA). After washing, the images were viewed and recorded using an Odyssey scanning system (Li-COR, USA).

\section{Statistical analysis}

All data presented were generated from triplicate samples. Results were calculated using a GraphPad Prism computer software and reported as mean $\pm \mathrm{SD}$. Comparison of differences between groups was determined by Student's $t$-test and analysis of variance (ANOVA). Statistical significance was defined as $\mathrm{P}<0.05$.

\section{Results}

\section{miRNAs differentially-expressed in 2- and 13-day-old mice hearts}

Using immunofluorescent staining for proliferation marker Ki67, we have demonstrated that the cardiomyocytes actively proliferated in 2-day-old neonate hearts but this rapidly ceases at 13 days. We estimated that $35.6 \% \pm 7.4 \%$ of 2-day-old cardiomyocytes were $\mathrm{Ki}-67^{+}$verses $3.8 \% \pm 3.5 \%$ in 13-day-old cardiomyocytes (Figures $1 A, B$ ). We also determined that the percentage of $\mathrm{pH} 3+$ cardiomyocytes was significantly decreased at day $13(9.5 \% \pm 3.4 \%$ vs. $2.7 \% \pm 2.2 \%$ at day 13 vs. day 2 , Figures $1 C, D)$. In this context, we examined the involvement of miRNAs in cardiac proliferation and differentiation. The global miRNA 

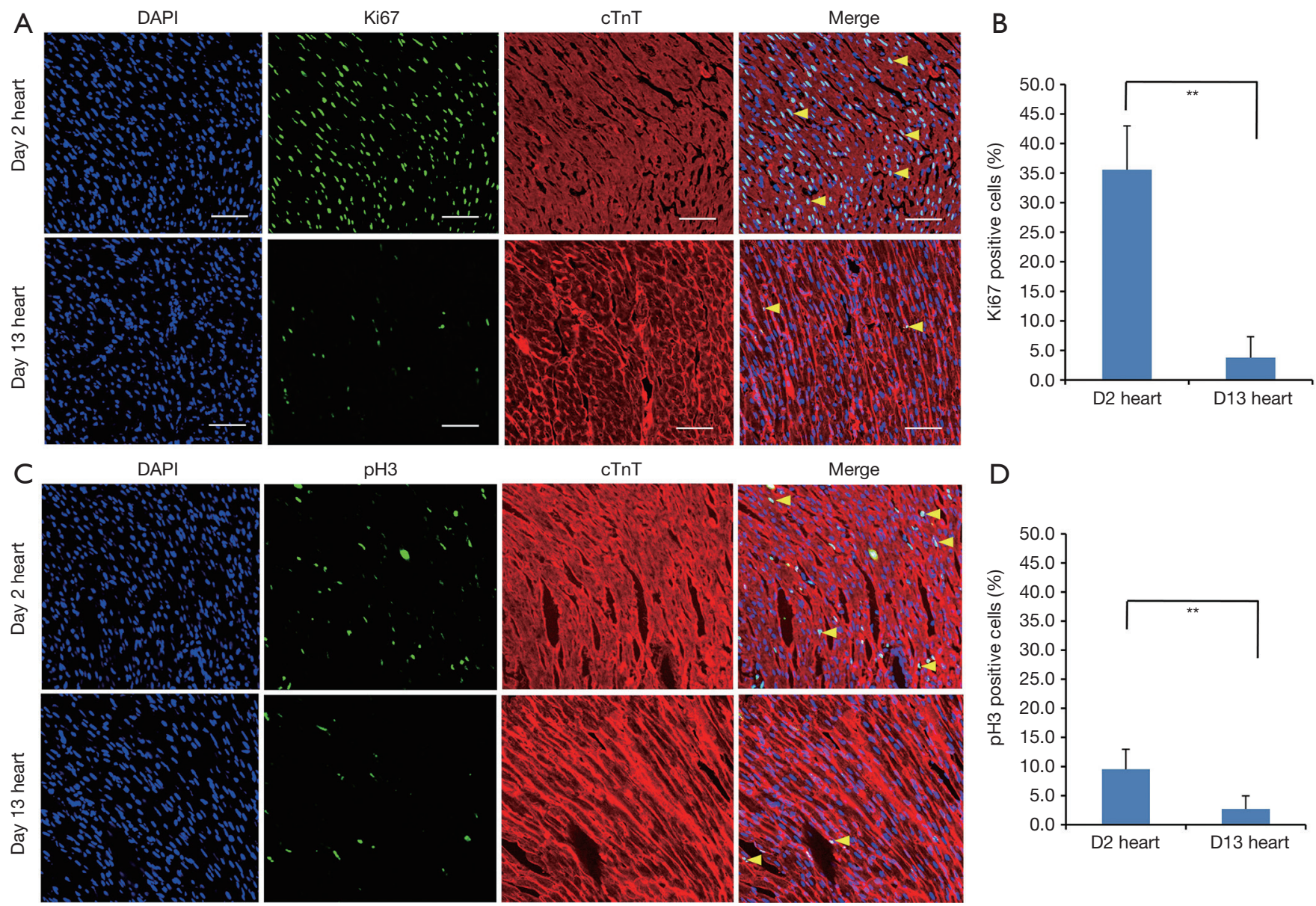

Figure 1 Cardiomyocyte proliferation in 2- and 13-day-old mouse hearts. Immunofluorescent staining showing (A) the presence of proliferating $\left(\mathrm{Ki}-67^{+}\right)$cardiomyocytes $\left(\mathrm{cTnT}^{+}\right)$at day 2 and day 13 (yellow arrows). Bar charts showing (B) the percentage of cardiomyocytes that were $\mathrm{Ki}-67^{+}$in 2 - and 13 -day-old hearts. Immunofluorescent staining showing (C) the presence of mitotic (pH3 ${ }^{+}$) cardiomyocytes $\left(\mathrm{cTnT} \mathrm{T}^{+}\right.$) at day 2 and day 13 (yellow arrows). Bar charts showing (D) the percentage of cardiomyocytes that were $\mathrm{pH} 3^{+}$in $2-$ and 13 -day-old hearts. Values are presented as mean \pm SD. scale bar: $50 \mu \mathrm{m}$. **, $\mathrm{P}<0.01$.

profiling of 2- and 13-day-old mice hearts were established and analyzed (http://fp.amegroups.cn/cms/atm.2019.08.681.pdf). The microarray identified six miRNAs that were differentially expressed (Figure $2 A$ ). We use RT-qPCR to validate the expression of these five miRNAs and determined that miR-1, miR-126 and miR-709 were significantly up-regulated in 13-day-old hearts compared with 2-day-old hearts. However, miR-322, miR-335 and miR-690 were not differentially expressed between the two time points. Moreover, miR-1 is the most significantly up- regulated miRNA in the 13-day-old heart (Figure 2B).

\section{Effects of miR-1 over-expression on cardiomyocyte} proliferation and viability

Cardiomyocytes were isolated from 2-day-old neonates and transfected with miR-1 mimics, mimics N.C. (control), miR-1 antagomir and antagomir N.C. (control) The effects of the transfections were examined by flow cytometry. The results revealed that the percentage of cardiomyocytes in G0/G1 phase of the miR-1 mimics group was significantly 
A

\begin{tabular}{|l|l|l|}
\hline & $\begin{array}{c}\text { Fold change } \\
\text { (D13 vs. D2) }\end{array}$ & $\begin{array}{c}\text { P-Value } \\
\text { (D13 vs. D2) }\end{array}$ \\
\hline mmu-miR-1 & 2.859817735 & 0.00279972 \\
\hline mmu-miR-1 & 3.025130413 & 0.00239976 \\
\hline mmu-miR-1 & 2.646074065 & 0.00439956 \\
\hline mmu-miR-1-126-3p & 3.566210267 & 0.00199980 \\
\hline mmu-miR-1-126-3p & 3.509109434 & 0.00139986 \\
\hline mmu-miR-1-126-3p & 3.587076897 & 0.00119988 \\
\hline mmu-miR-322 & -3.503680635 & 0.00199980 \\
\hline mmu-miR-322 & -3.676864041 & 0.00039996 \\
\hline mmu-miR-322 & -4.082049708 & 0.00039996 \\
\hline mmu-miR-335 & -2.582242918 & 0.00439956 \\
\hline mmu-miR-335 & -2.263860155 & 0.01059894 \\
\hline mmu-miR-335 & -2.688264607 & 0.00359964 \\
\hline mmu-miR-690 & 4.472847372 & 0.00039996 \\
\hline mmu-miR-690 & 6.061224178 & 0.00019998 \\
\hline mmu-miR-690 & 5.979517650 & 0.00019998 \\
\hline mmu-miR-709 & 4.795312153 & 0.00039996 \\
\hline mmu-miR-709 & 5.191795790 & 0.00019998 \\
\hline mmu-miR-709 & 5.854211493 & 0.00019998 \\
\hline
\end{tabular}

B

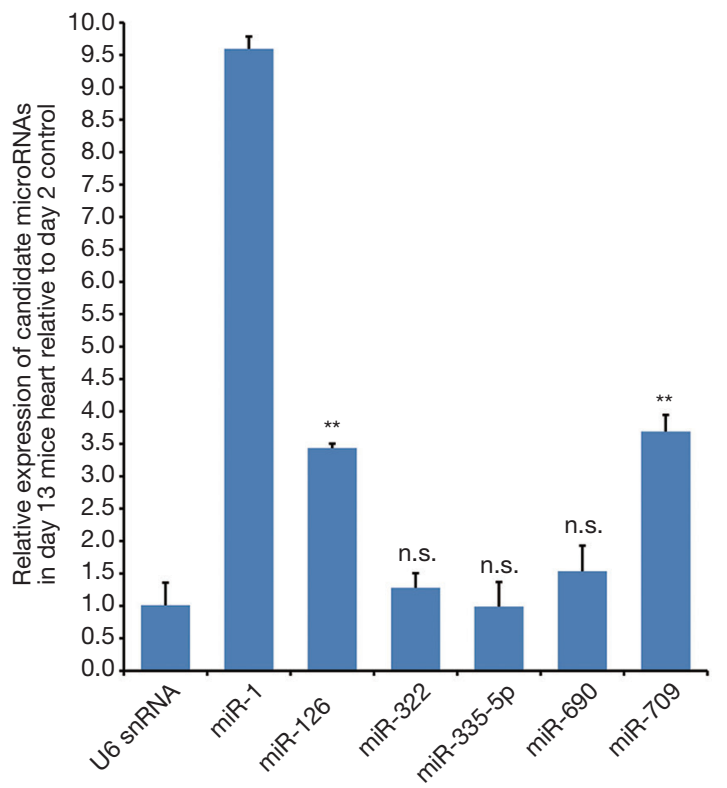

Figure 2 microRNAs differentially expressed in 2- and 13-day-old mouse hearts. (A) Six significant differentially expressed miRNAs were identified in 2 and 13-day-old mouse hearts (performed in tri-plicate, $\mathrm{P}<0.05$ ). (B) Stem Loop RT-qPCR analysis validated the miRNA microarray data that five miRNAs differentially expressed. Expression values are presented as mean $\pm \mathrm{SD}$. ${ }^{* *}, \mathrm{P}<0.01$. ns, no significance.

increased compared with that in mimics N.C. group. This was accompanied by a concomitant decrease of cell number in the $\mathrm{S}$ phase. Moreover, the percentage of cardiomyocytes in G0/G1 phase for the miR-1 antagomir group was significantly decreased compared with the antagomir N.C. group (Figures $3 A, B)$. These data suggest that overexpression of miR-1 in 2-day-old cardiomyocytes reduces the number of these cells in $\mathrm{S}$ phase but increases them in the G0/G1 phase.

We also investigated the effects of miR-1 on cardiomyocyte viability. MTT assays were performed after the cells were transfected with miR-1 mimics or mimics N.C. These results show that cardiomyocyte viability was significantly decreased following miR-1 mimics compared with mimics N.C. and also without any treatment (Figure $3 C, \mathrm{P}<0.05$ ). The results suggest that miR-1 reduces cardiomyocyte viability.

The effects of miR-1 on cardiomyocyte proliferation were further investigated by BrdU-incorporation assay. We observed that the cell number was significantly decreased following transfection with miR-1 mimics (Figures 3D,E). Furthermore, the proportion of $\mathrm{BrdU}^{+}$cardiomyocytes was significantly decreased compared with cardiomyocytes transfected with mimics N.C. (Figures $3 F, G$ ). These results indicate that over-expression of miR-1 inhibits cardiomyocyte proliferation.

\section{Predicated target genes regulated by $\mathrm{miR}-1$}

In order to understand how miR-1 inhibited cardiomyocyte proliferation, we searched available databases for potential target genes relevant to the biological process using computational online software. We found that miR-1 could potentially bind with the 3'UTR sites of FGF4, Gas2L1, IGF-1, Frs2, FoxP1 Cdc14a, CCND2, CCND1 and CDC14a mRNAs, which contains highly conserved miR-1 seed-matching sequences. FGF-4, IGF-1, CCND1 and Cdc14a encode the for peptides: fibroblast growth factor 4, insulin-like growth factor 1, cyclinD1 and CDC14 cell division cycle 14 homolog A, respectively. We determined that Frs2, FoxP1, IGF-1 and CCND1 expressions were significantly down-regulated compared with expression of these genes in cardiomyocytes transfected with negative control oligonucleotides at $48 \mathrm{hr}$ after miR-1 mimics were transfected into 2-day-old cardiomyocytes (Figure 4A).

The question that we wanted address was whether the predicated target genes of miR-1 were also down-regulated in the 13-day-old cardiomyocytes (developmental stage 

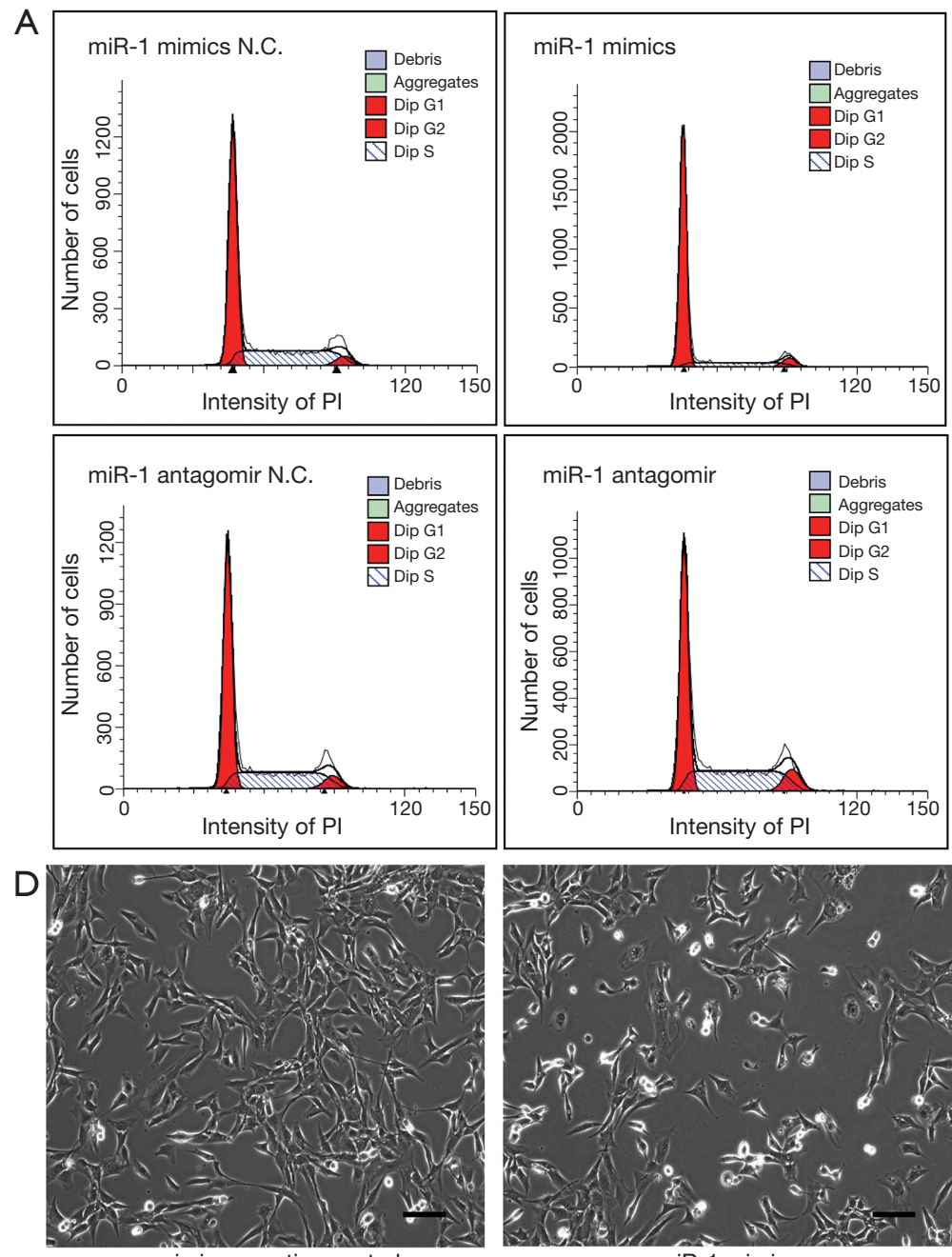

mimics negative control
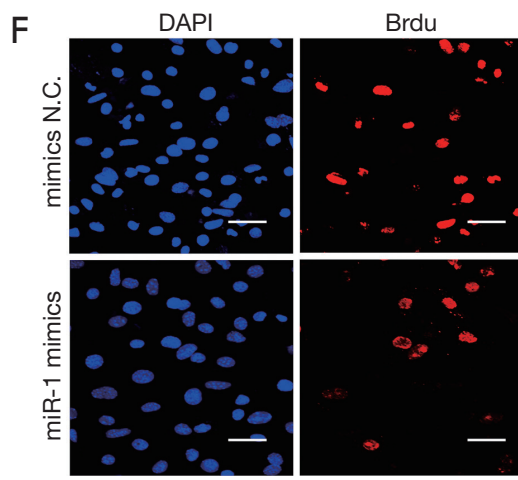

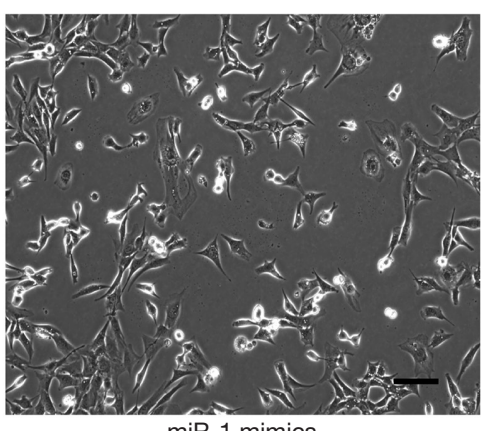

miR-1 mimics
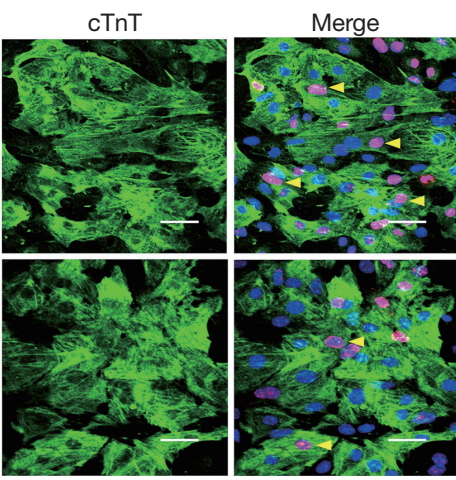

B

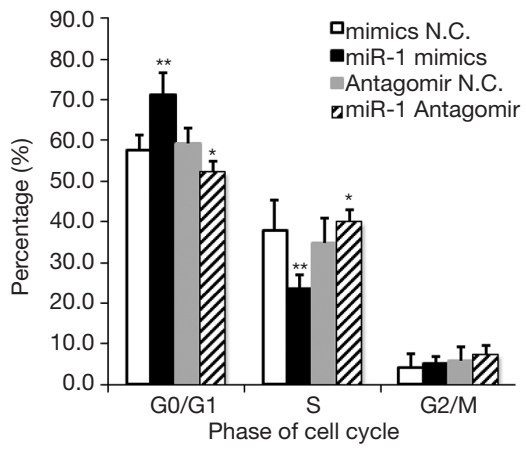

C
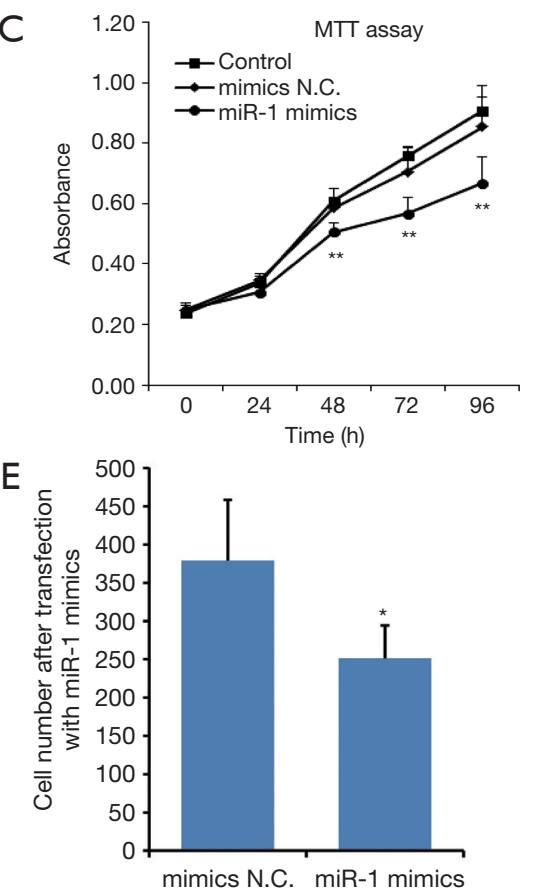

G

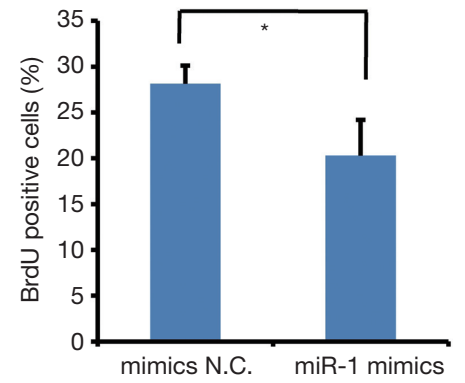

Figure 3 miR-1 inhibit cell cycle, proliferation and viability of neonatal cardiomyocytes. Cell cycle distribution (A) of cardiomyocytes after transfection with miR-1 mimics, mimics negative control (N.C), miR-1 antagomir and antagomir negative control. The cells transfected with miR-1 mimics showed an increased in G0/G1 phase and a decrease in S-phase compare with the mimics control (B). The height of the columns represents the viability of cardiomyocytes as determined by MTT assay (C). The cell density and number were determined under bright fields of microscope (D and E). Scale bar $=20 \mu \mathrm{m}$. Cell proliferation was analyzed by BrdU-incorporation assay (F, scale bar $=50 \mu \mathrm{m}$ ). Quantification of $\mathrm{BrdU}^{+}$cardiomyocytes is presented as the percentage of $\mathrm{BrdU}^{+}$cells out of the total number of $\mathrm{DAPI}^{+}$cells $(\mathrm{G})$. The values are presented as mean $\pm \mathrm{SD}$ from three experiments. *, $\mathrm{P}<0.05 ;{ }^{* *}, \mathrm{P}<0.01$. ns, no significance. 

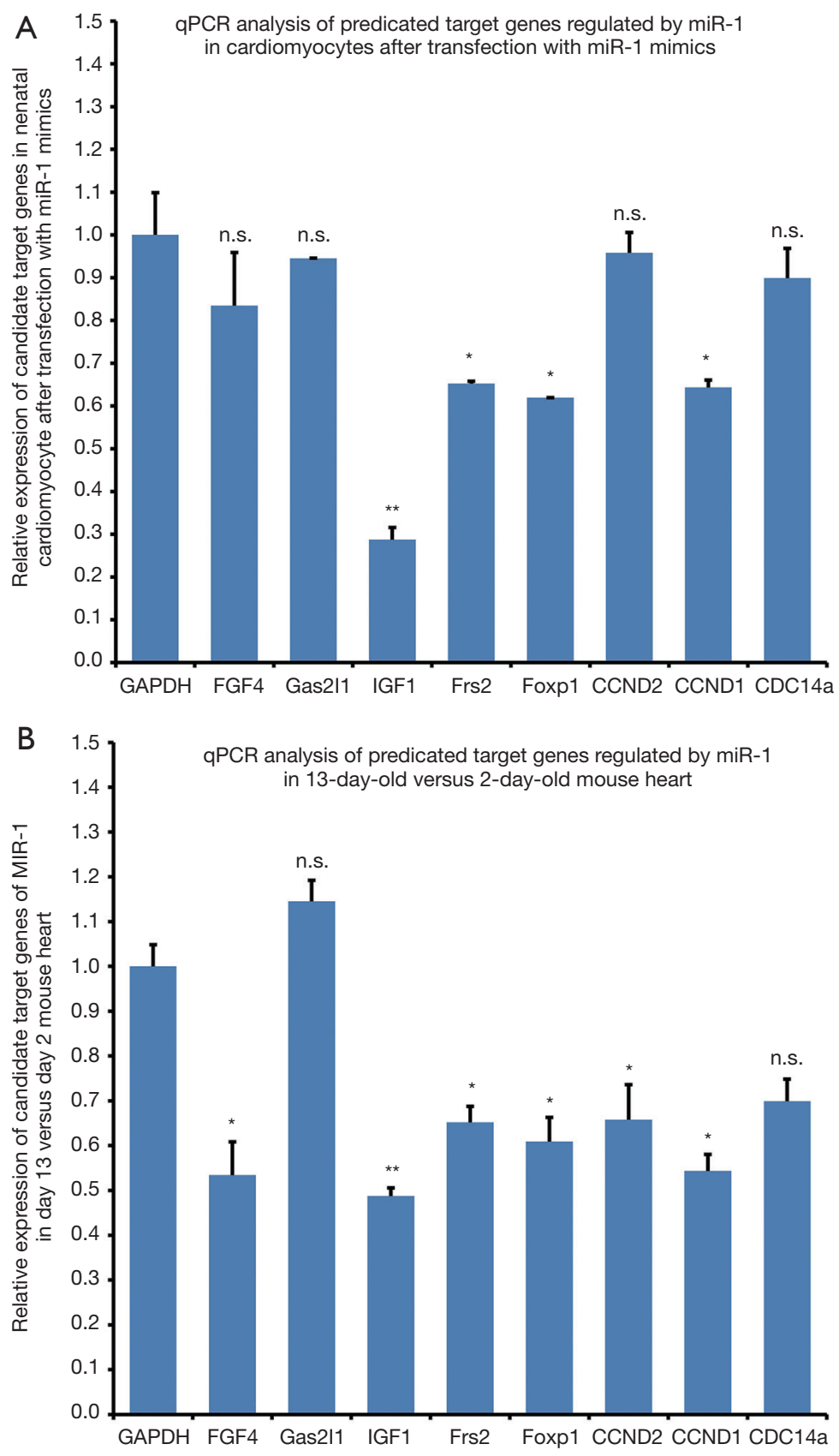

Figure 4 RT-qPCR assays. Validation of target genes predicated to be regulated by miR-1 in neonatal cardiomyocytes after transfection with miR-1 mimics (A), and also in 2- and 13-day-old hearts (B). Relative expression values are presented as mean $\pm \mathrm{SD}$. ${ }^{*} \mathrm{P}<0.05,{ }^{* *} \mathrm{P}<0.01$. ns, no significance.

when cardiomyocytes have almost stopped dividing). We used RT-qPCR to establish the mRNA abundance of these genes in 2-day-old and 13-day-old mice hearts to elucidate whether there was a correlation between the expressions of these genes in vivo with our in vitro results. We established that FGF4, IGF-1, Frs2, FoxP1, CCND1 and CDC14a expressions were significantly down-regulated in the 13-day-old heart compared with 2-day-old mouse heart (Figure 4B). These results suggest that the rapid decline in cardiomyocyte proliferation during the post-natal period is 
associated with a corresponding decrease in miR-1 targeted genes expression (genes that are associated with the cell cycle). Furthermore, IGF-1, Frs2, FoxP1 and CCND1 expression were down-regulated in both miR-1 transfected neonatal and adult cardiomyocytes.

\section{Identification of $m R N A$ Targets for $m i R-1$}

We attempted to confirm that IGF-1, Frs2, FoxP1 and CCND1 3'UTR (Figure 5A) were direct targets of miR-1. pmirGLO-UTR vectors (Figure 5B) containing the miR-1 binding sites were constructed to perform reporter assays and the pmirGLO empty vector without 3'UTR was set as a control. All the four constructs were validated by restriction enzyme digestion and sequencing. The luciferase activity of the reporter vectors was assayed $48 \mathrm{hr}$ after cotransfection of the reporter construct with miR-1 mimics or negative control in 2-day-old cardiomyocytes. Following the co-transfection of neonatal cardiomyocytes with miR-1 mimics, the relative luciferase activity of pmirGLO-IGF1 3'UTR and pmirGLO-CCND1 3'UTR were significantly suppressed by $52 \%$, respectively (Figure $5 C$ ) in comparison to the co-transfection with the negative control. In addition, the relative luciferase activity did not change when cotransfected with miR-1 and pmirGLO empty vector, indicating that IGF1 3 'UTR and CCND1 3'UTR were direct targets of miR-1.

\section{IGF-1 and CCND1 expression in cardiomyocytes over- expressing $m i R-1$}

Since miR-1 can inhibit G1/S transition, we decided to examine the effects of miR-1 over-expression on CCND1 and IGF1 which regulate cardiac cell cycle and growth respectively. Using immunofluorescent staining, we examined IGF-1 and CCND1 expression in 2-dayold cardiomyocytes $48 \mathrm{hr}$ after miR-1 mimics or mimics N.C. transfection. Immunofluorescence staining showed that CCND1 expression was repressed in cardiomyocytes transfected with miR-1 mimics (Figures 6A,B). However, IGF-1 expression was unaffected when cardiomyocytes were transfected with miR-1 mimics (Figure 6C). Western blotting was also performed and the results revealed that CCND1 expression was significantly down-regulated in cardiomyocytes $48 \mathrm{hr}$ after miR-1 mimics transfection as compared with miR-1 mimics compared with miR-1 mimics N.C. (negative control). However, IGF-1 expression was unaffected by the miR-1 mimics (Figure 6D). The data suggests that over-expressing miR-1 mimics represses CCND1 expression.

\section{IGF-1 and CCND1 expression in 2- and 13-day-old bearts}

It has been reported that IGF1 is an important cytokine for cell cycle progression and growth. In cardiomyocytes, it regulates cell proliferation, apoptosis and other biological processes. CCND1 is a key regulator of cell cycle, so we decided to examine IGF1 and CCND1 expression in 2- and 13-day-old hearts. We determined that CCND1 expression was down-regulated in 13 - verses 2-day-old hearts (Figure $7 A, B$ ). However, the immunofluorescent staining did not show any obvious difference in IGF-1 expression between the two time points (Figure 7C).

\section{Bioinformatics analysis of differentially expressed $m R N A$ and $m R N A$ targets of $m i R-1$}

We have previously reported that the transcripts most significantly down-regulated in 13-day-old cardiomyocytes, were associated with the "cell cycle" as revealed by bioinformatics analysis (13). In addition, above we have demonstrated that is miR-1 was down-regulated in 13-dayold hearts (Figure 2B). These findings allowed us to narrow down our search for differentially expressed mRNA and the predicated mRNA targets of miR-1 that were associated with cardiomyocyte proliferation. Comparison of the two lists for overlapping genes revealed that IGF1 and CCND1 transcripts were down-regulated and simultaneously are amongst the predicated mRNA targets of miR-1 (Table S2). Bioinformatics analysis of the differentially expressed mRNA (13) and mRNA targets of miR-1 (http:// fp.amegroups.cn/cms/atm.2019.08.68-2.pdf) further emphasized that IGF1 and CCND1 were targets of miR-1 in neonatal cardiomyocyte.

\section{Discussion}

Cardiomyocyte growth and differentiation are precisely regulated and involves the cell cycle. The different phases of the cell-cycle are orchestrated by positive and negative regulatory proteins. Hence, it is important to elucidate the proteins and miRNAs that control the cardiomyocyte cell-cycle progression and arrest. It will potentially provide tools for resetting the growth-arrested adult cardiomyocytes back into proliferation and repair degenerative heart conditions. It is generally thought that 

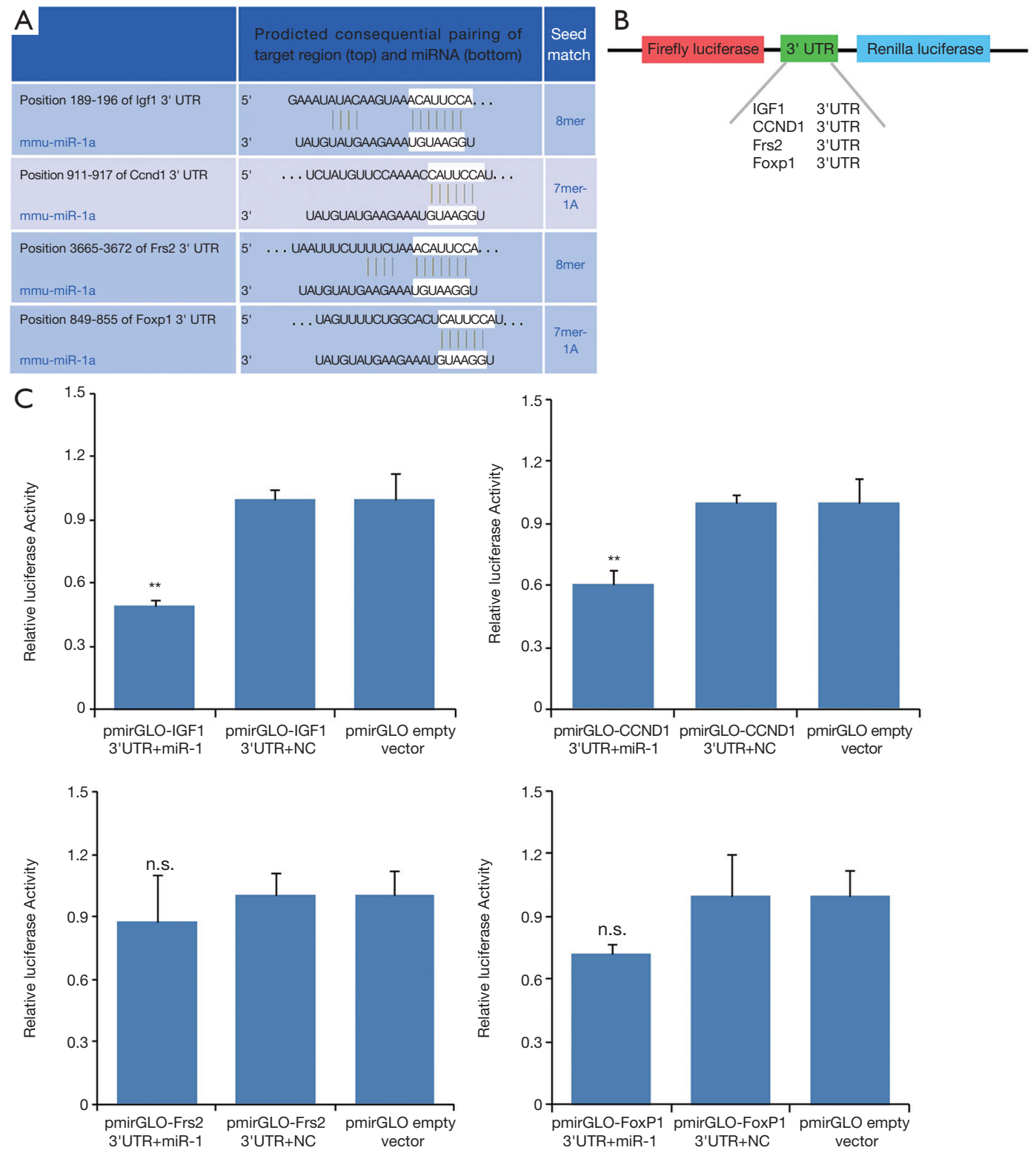

Figure 5 Ability of miR-1 to target the 3'UTR of IGF1 and CCND1 mRNA in neonatal cardiomyocytes. (A) Alignment of 5' seeding region within miR-1 mature sequence and the putative binding sites within the 3'UTR region of IGF1, CCND1, Frs2 and FoxP1-mRNAs using TargetScan database. (B) Construct luciferase reporter containing the predicted binding sites within the 3'UTR of IGF1, CCND1, Frs2 and FoxP1 mRNAs into downstream of the firefly luciferase gene of pmirGLO vector. (C) Dual-Glo Luciferase reporter assays revealed reduced luciferase activity after co-transfection of pmirGLO-IGF1 3'UTR vector and pmirGLO-CCND1 3'UTR vector, respectively, with miR-1 mimics. The results were compared with luciferase activities from cardiomyocytes transfected with miR-1 mimics N.C. and pmirGLO empty vectors. The data are presented as mean \pm SD from triplicated experiments. ${ }^{* *}, \mathrm{P}<0.01$ vs. miR-1 mimics $\mathrm{NC}$ or pmirGLO empty vectors. 

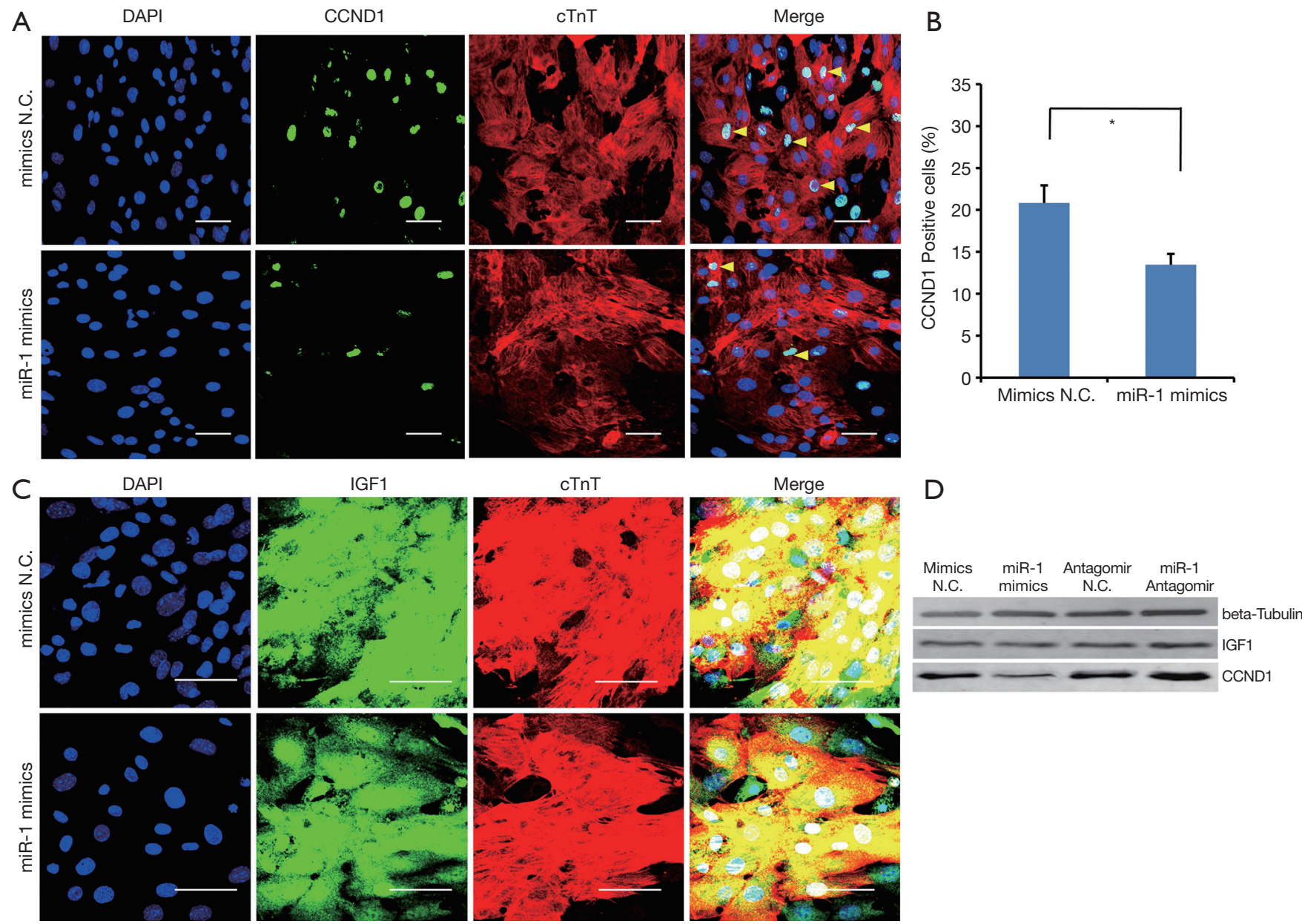

Figure 6 Over-expression of miR1 decreases CCND1, but not IGF1, protein expression. Immunocytofluorescent staining revealed that CCND1 expression was significantly inhibited in cardiomyocytes following miR-1 mimics transfection (A and B). In contrast, no significant different difference in IGF1 expression in c'TnT+ neonatal cardiomyocytes transfected with miR-1 mimics (C). Western blotting also confirmed that CCND1 expression was significantly down-regulated in cardiomyocytes after transfection with miR-1 mimics compared to the negative control (D). *, $\mathrm{P}<0.05$. Scale bar $=50 \mu \mathrm{m}$.

adult cardiomyocytes in mammals are incapable of selfrenewable, as these cells are growth arrested shortly after birth. Neonatal cardiomyocytes will stop dividing at around 7 days after birth in mice and 3-4 days in rats (5). In these hearts, $85 \%$ of the cardiomyocytes are arrested in G0/G1 phase as determined with flow cytometry. For humans, cardiomyocytes proliferation begins to stop at 3-6 month after birth, with the cells arrested in the G0/G1 phase (14-16). The G0/G1 phase of cell cycle is regulated by cyclins that include cyclin C, D and E. Cyclin D1 plays an important role in G1/S phase transition and proliferation of cells (17) but it could not escape from being down-regulated in the mouse heart after the first week of birth (18).
Cardiovascular disease is one of the leading causes for death in both developed and developing countries (18). Abnormal expression of key genes and signaling transduction are involved in the pathogenesis of cardiovascular diseases. Amongst this, miRNAs are newly found molecules that regulate about $30 \%$ of the total gene expression in the human genome, at the post-transcriptional level (19). Numerous studies have demonstrated that miRNAs play an important role apoptosis, cell growth and differentiation, implying that miRNAs are important regulators of development and physiological processes. Hence, miRNAs dysfunction and their roles in fine tuning of mRNA translation may pathologically lead to various 
A

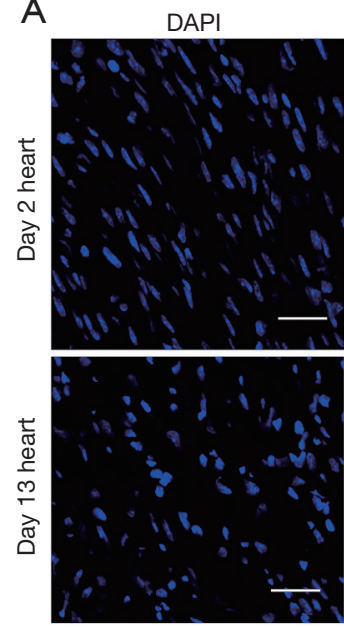

C
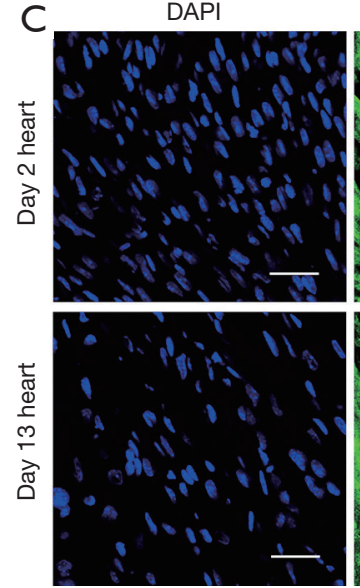

CCND1

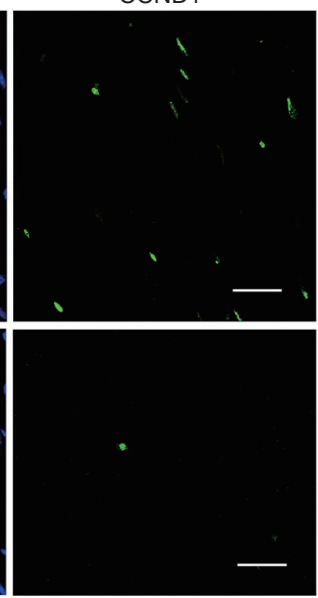

IGF1
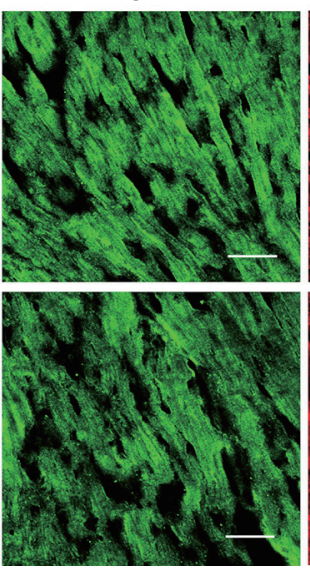

cTnT

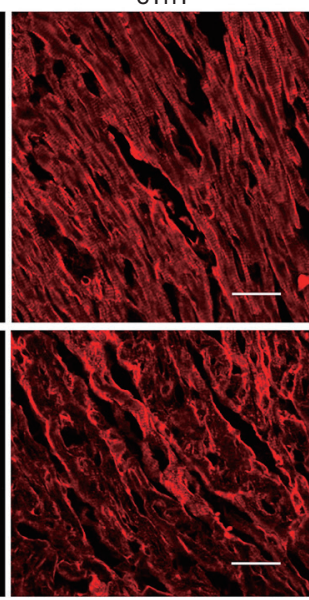

cTnT
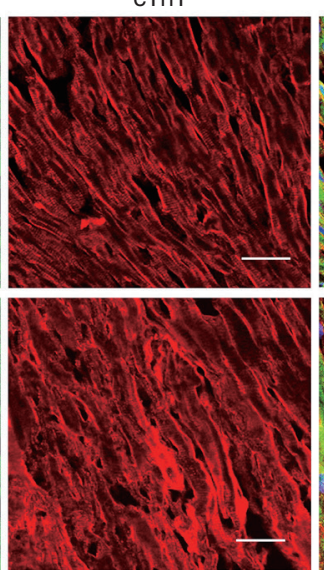

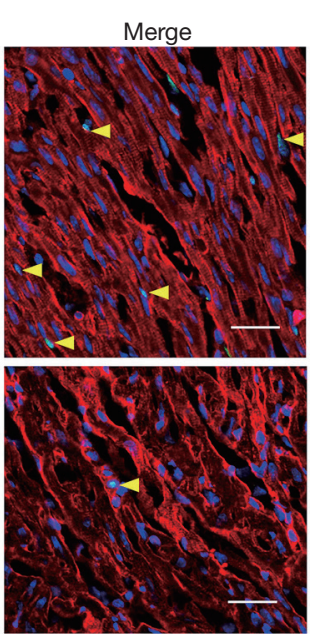

B

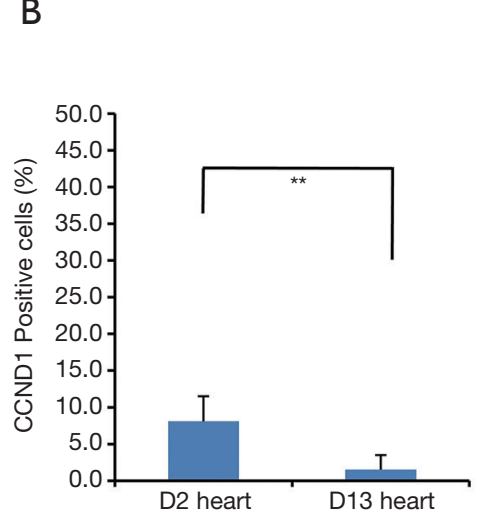

Figure 7 IGF-1 and CCND1 expression in 2- and 13-day-old hearts. The normal expression patterns of IGF1 and CCND1 were investigated in 2- and 13-day-old mouse hearts. Immunocytofluorescent staining revealed that CCND1 expression is down-regulated in 13-day-old hearts (A and B). In contrast, no significant change in IGF1 expression between 2- and 13-day-old mouse hearts (C). Values are presented as mean $\pm \mathrm{SD}$. scale bar $=50 \mu \mathrm{m}$. ${ }^{* *}, \mathrm{P}<0.01$. ns, no significance.

diseases (20). Tissue-specific expression is an important feature of miRNAs and miR1, miR126-3P, miR-133, miR499 and Let-7 are abundantly expressed in the heart $(21,22)$. It has been reported that miR-1 is specifically expressed in cardiac and skeletal muscle tissues (23-26). In this study, we have validated that miR-1 is expressed more intensely in the 13- than 2-day-old hearts. In 13-day-old hearts, most of the cardiomyocytes have stopped dividing and have entered into "irreversible" growth arrest.

We examined cardiomyocyte viability, cell cycle progression and BrdU incorporation following miR-1 mimics over-expression in 2-day-old cardiomyocytes. MTT assay was used to assess cell viability. The assay is based on succinate dehydrogenase present in mitochondria of living cells that can reduce exogenous MTT to Formazan. Cells that are dead do not have this function so will not produce Formazan. Our results revealed that cardiomyocyte viability was significantly decreased following miR-1 mimics transfection. Our flow cytometry analysis, also indicated that miR-1 increased the number of cardiomyocytes in the G0/ G1 phase but decreased the number of cells in $S$ phase. This implies that these cardiomyocytes were blocked at G1/S transition. In support, the proportion of $\mathrm{BrdU}^{+}$cardiomyocytes in culture was significantly decreased following transfection with miR-1 as compared with mimics N.C (control). We concluded that miR-1 inhibited cardiomyocyte viability, G1/S cell cycle transition, and proliferation.

We predicted that IGF1 and CCND1 were candidate 
target genes regulated by miR-1. In addition, our RTqPCR and the luciferase reporter assays demonstrated that miR-1 could suppress the expression of these two genes at the mRNA level. Our immunocytofluorescent staining and western blot results also confirmed that miR-1 inhibited CCND1 expression at the protein level. In support, we have shown that miR-1 was strongly expressed in the 13-dayold (growth arrested) heart, while CCND1 expression was correspondingly downregulated when compared with 2-day-old (proliferative) heart.

This study has identified miR-1 as an effective inhibitor of CCND1 expression during cardiomyocyte proliferation in the mouse neonatal heart. We have determined that miR-1 could regulate IGF1 expression at the mRNA level but could not demonstrate a corresponding change at the protein level, when cardiomyocytes were transfected with the miR-1 mimics. Furthermore, immunofluorescent staining of 2-day-old and 13-day-old hearts revealed that the cardiomyocytes at both developmental stages embodied the same level of IGF1 expression. Lastly, we discovered that miR-1 antagomir transfected into cardiomyocytes did not exert a positive proliferative effect on cardiomyocytes, which was inconsistent with our expectation. Despite this inconsistency, our study is the first to report miR1's ability to inhibit cardiomyocytes proliferation via the inhibition of CCND1 expression. Our findings also suggest that if we could identify a method to effectively inhibit miR-1 expression or stimulate CCND1 expression, then a novel therapeutic tool could be developed to reactivate cardiomyocyte proliferation after heart injury.

\section{Conclusions}

In this study, we have demonstrated that miR-1 was the most significantly upregulated miRNA in 13-day-old mouse heart compared with 2-day-old heart. We also revealed that miR-1 could repress cardiomyocyte G1/S phase transition, proliferation and viability. These results suggest that miR-1 plays an important role in inhibiting cardiomyocyte proliferation in the developing neonatal mouse heart by directly suppressing the cell-cycle regulator, CCND1.

\section{Acknowledgments}

Funding: This study was supported by General Research Fund (14100315). Guangdong Science and Technology Bureau International Science and Technology Collaboration Program (20130501c).

\section{Footnote}

Conflicts of Interest: The authors have no conflicts of interest to declare.

Ethical Statement: All experimental protocol used in this study was approved by the Chinese University of Hong Kong Animal Care and User Committee and Department of Health (Ref No.: 13-2 in DH/HA\&P/8/2/1 Pt.28). The authors are accountable for all aspects of the work in ensuring that questions related to the accuracy or integrity of any part of the work are appropriately investigated and resolved.

\section{References}

1. Huang Y, Harrison MR, Osorio A, et al. Igf Signaling is Required for Cardiomyocyte Proliferation during Zebrafish Heart Development and Regeneration. PLoS One 2013;8:e67266.

2. Zhang $\mathrm{R}$, Han $\mathrm{P}$, Yang $\mathrm{H}$, et al. In vivo cardiac reprogramming contributes to zebrafish heart regeneration. Nature 2013;498:497-501.

3. Tang MK, Kindler PM, Cai DQ, et al. Heart-type fatty acid binding proteins are upregulated during terminal differentiation of mouse cardiomyocytes, as revealed by proteomic analysis. Cell Tissue Res 2004;316:339-47.

4. Liu Y, Tang MK, Cai DQ, et al. Cyclin I and p53 are differentially expressed during the terminal differentiation of the postnatal mouse heart. Proteomics 2007;7:23-32.

5. Porrello ER, Mahmoud AI, Simpson E, et al. Transient Regenerative Potential of the Neonatal Mouse Heart. Science 2011;331:1078-80.

6. Eulalio A, Mano M, Ferro MD, et al. Functional screening identifies miRNAs inducing cardiac regeneration. Nature 2012;492:376-81.

7. Wahlquist C, Jeong D, Rojas-Munoz A, et al. Inhibition of miR-25 improves cardiac contractility in the failing heart. Nature 2014;508:531-5.

8. Feng Y, Yu X. Cardinal roles of miRNA in cardiac development and disease. Science China Life Sciences 2011;54:1113-20.

9. Tang Y, Liu D, Zhang L, et al. Quantitative Analysis of miRNA Expression in Seven Human Foetal and Adult Organs. PLoS One 2011;6:e28730.

10. Pritchard CC, Cheng HH, Tewari M. MicroRNA profiling: approaches and considerations. Nat Rev Genet 2012;13:358-69. 
11. Feldman MY. Reactions of nucleic acids and nucleoproteins with formaldehyde. Prog Nucleic Acid Res Mol Biol 1973;13:1-49.

12. Chen E, Tang MK, Yao Y, et al. Silencing BRE Expression in Human Umbilical Cord Perivascular (HUCPV) Progenitor Cells Accelerates Osteogenic and Chondrogenic Differentiation. PLoS One 2013;8:e67896.

13. Gan J, Sonntag H-J, Tang Mk, et al. Integrative Analysis of the Developing Postnatal Mouse Heart Transcriptome. PLoS One 2015;10:e0133288.

14. Di Stefano V, Giacca M, Capogrossi MC, et al. Knockdown of Cyclin-dependent Kinase Inhibitors Induces Cardiomyocyte Re-entry in the Cell Cycle. J Biol Chem 2011;286:8644-54.

15. Cheng RK, Asai T, Tang H, et al. Cyclin A2 Induces Cardiac Regeneration After Myocardial Infarction and Prevents Heart Failure. Circ Res 2007;100:1741-8.

16. Nakajima K, Inagawa M, Uchida C, et al. Coordinated regulation of differentiation and proliferation of embryonic cardiomyocytes by a jumonji (Jarid2)-cyclin D1 pathway. Development 2011;138:1771-82.

17. Tane S, Kubota M, Okayama H, et al. Repression of Cyclin D1 Expression Is Necessary for the Maintenance of Cell Cycle Exit in Adult Mammalian Cardiomyocytes. J Biol Chem 2014;289:18033-44.

18. Go AS, Mozaffarian D, Roger VL, et al. Heart Disease and Stroke Statistics-2014 Update: A Report From the American Heart Association. Circulation 2014;129:e28-e292.

Cite this article as: Gan J, Tang FM, Su X, Lu G, Xu J, Lee HS, Lee KK. microRNA-1 inhibits cardiomyocyte proliferation in mouse neonatal hearts by repressing CCND1 expression. Ann Transl Med 2019;7(18):455. doi: 10.21037/ atm.2019.08.68
19. Lewis BP, Burge CB, Bartel DP. Conserved Seed Pairing, Often Flanked by Adenosines, Indicates that Thousands of Human Genes are MicroRNA Targets. Cell 2005;120:15-20.

20. Alvarez-Garcia I, Miska EA. MicroRNA functions in animal development and human disease. Development 2005;132:4653-62.

21. Cheng Y, Ji R, Yue J, et al. MicroRNAs Are Aberrantly Expressed in Hypertrophic Heart. Am J Pathol 2007;170:1831-40.

22. Sluijter JP, van Mil A, van Vliet P, et al. MicroRNA-1 and -499 Regulate Differentiation and Proliferation in HumanDerived Cardiomyocyte Progenitor Cells. Arterioscler Thromb Vasc Biol 2010;30:859-68.

23. Li Q, Song XW, Zou J, et al. Attenuation of microRNA-1 derepresses the cytoskeleton regulatory protein twinfilin-1 to provoke cardiac hypertrophy. J Cell Sci 2010;123:2444-52.

24. Zhao Y, Ransom JF, Li A, et al. Dysregulation of Cardiogenesis, Cardiac Conduction, and Cell Cycle in Mice Lacking miRNA-1-2. Cell 2007;129:303-17.

25. Liu L, Yuan Y, He X, et al. MicroRNA-1 upregulation promotes myocardiocyte proliferation and suppresses apoptosis during heart development. Mol Med Rep 2017;15:2837-42.

26. Kwon C, Han Z, Olson EN, et al. MicroRNA1 influences cardiac differentiation in Drosophila and regulates Notch signaling. Proc Natl Acad Sci U S A 2005;102:18986-91. 
Table S1 Primers of genes used in this context

\begin{tabular}{|c|c|}
\hline Name & Sequence (5' to $\left.3^{\prime}\right)$ \\
\hline miR1-RT & 5'-GTCGTATCCAGTGCAGGGTCCGAGGTATTCGCACTGGATACGACATACAT-3' \\
\hline miR1-F & 5'-GCGGCGGTGGAATGTAAAGAAG-3' \\
\hline miR126-RT & 5'-GTCGTATCCAGTGCAGGGTCCGAGGTATTCGCACTGGATACGACCGCATT-3' \\
\hline miR126-F & 5'-GCGGCGTCGTACCGTGAGTAA-3' \\
\hline MiR322-RT & 5'-CTCAACTGGTGTCGTGGAGTCGGCAATTCAGTTGAGTCCAAAAC-3' \\
\hline MiR322-F & 5'-ACACTCCAGCTGGGCAGCAGCAATTCATGT-3' \\
\hline miR3355-RT & 5'-GTCGTATCCAGTGCAGGGTCCGAGGTATTCGCACTGGATACGACACATTT-3' \\
\hline miR3355-F & 5'-CGGCGGTCAAGAGCAATAACG-3' \\
\hline miR690-RT & 5'-GTCGTATCCAGTGCAGGGTCCGAGGTATTCGCACTGGATACGACTTTGGT-3' \\
\hline miR690-F & 5'-TGCGGAAAGGCTAGGCTCAC-3' \\
\hline miR709-RT & 5'-GTCGTATCCAGTGCAGGGTCCGAGGTATTCGCACTGGATACGACTCCTCC-3' \\
\hline miR709-F & 5'-TTCCTGGAGGCAGAGGCA-3' \\
\hline miRU6-RT & 5'-GTCGTATCCAGTGCAGGGTCCGAGGTATTCGCACTGGATACGACCATCCT-3' \\
\hline miRU6-F & 5'-TTCCTTGGCCCCTGCGCA-3' \\
\hline mmu-miR-1 mimics & 5'-UGGAAUGUAAAGAAGUAUGUAU-3', 5'-ACAUA CUUCUUUACA UUCCAUU-3' \\
\hline mimics n.c. & 5'-UUCUCCGAACGUGUCACGUTT-3', 5'-ACGUGACACG UUCGG AGAATT-3' \\
\hline mmu-miR-1 antagomiR & 5'-AUACAUACUUCUUUA CAUUCCA-3' \\
\hline antagomiR n.c. & 5'-UUGUACUACACAAAA GUACUG-3' \\
\hline 1 FGF4-F & 5'-TGGTGAGCATCTTCGGAGTG-3' \\
\hline 1 FGF4-R & 5'-GCGTTGTAGTTGTTGGGCAG-3' \\
\hline 2 Gas2l1-F & 5'-ACGCCCAATGACCTTCGAA-3' \\
\hline 2 Gas2l1-R & 5'-CATCACGTGACTCCTCAGCA-3' \\
\hline 3 IGF1-F & 5'-GCTGGTGGATGCTCTTCAGT-3' \\
\hline 3 IGF1-R & 5'-TCCGGAAGCAACACTCATCC-3' \\
\hline 4 Frs2-F & 5'-TTGGAGACGCTTCATCCCAC-3' \\
\hline 4 Frs2-R & 5'-ACTTGTTCCTCAGCCACGAG-3' \\
\hline 5 Foxp1-F & 5'-TGACGACTTCCCAGCCTTTC-3' \\
\hline 5 Foxp1-R & 5'-AGGTGGGTCATCATGGCTTG-3' \\
\hline 6 CDK9-F & 5'-CTGCGATGAGGTCACCAAGT-3' \\
\hline 6 CDK9-R & 5'-TCAAGGCTGTGATGGGGAAC-3' \\
\hline 7 CCND2-F & 5'-TACCTCCCGCAGTGTTCCTA-3' \\
\hline 7 CCND2-R & 5'-GCCAAGAAACGGTCCAGGTA-3' \\
\hline 8 CCND1-F & 5'-TGCCGAGAAGTTGTGCATCT-3' \\
\hline 8 CCND1-R & 5'-GCTTGTTCTCATCCGCCTCT-3' \\
\hline $9 \mathrm{CDC} 14 \mathrm{a}-\mathrm{F}$ & 5'-GGAAAAGAGCAAACGCAGCA-3' \\
\hline 9 CDC14a-R & 5'-GTCAAGGACGGTGAGGTTGT-3' \\
\hline 10 mGAPDH & 5'-AAATACGGACTGCAGCCCTC-3' \\
\hline 10 mGAPDH & 5'-ATCCGTTCACACCGACCTTC-3' \\
\hline 1clone-IGF1-F & 5'-GCGAGCTCAGAAAATGCCACATCACCGC-3' \\
\hline 1clone-IGF1-R & 5'-GCTCTAGAAGCTACGTGGGAAGAGGTGA-3' \\
\hline 2clone-Frs2-F & 5'-GCGAGCTCAACCCTGCGTCCTCAATGTT-3' \\
\hline 2clone-Frs2-R & 5'-GCTCTAGAGCCAACCCAAGATTAACCTGC-3' \\
\hline 3clone-Foxp1-F & 5'-GCGAGCTCCAGCGCTGCATGTTGATACC-3' \\
\hline 3clone-Foxp1-R & 5'-GCTCTAGACGTGCGTTTCTCTCTTTCCTTा-3' \\
\hline 4clone-CCND1-F & 5'-GCGAGCTCTAGTGGTCTCATGGCGTGTG-3' \\
\hline 4clone-CCND1-R & 5'-GCTCTAGAGGTGGTGCGAGAACAGAGTT-3' \\
\hline
\end{tabular}

Table S2 The overlapped gene list between down-regulated mRNA and mRNA targets of miR-1

\section{Gene name:}

Acer2, Fubp1, Pde4dip, Alpl, Glcci1, Palm2, Ankrd23, Gpr125, Plekho2, Azin1, Hic2, Prl, BC024479, Hmgcr, Ptprd, Bcl11a, Hnrnpa1, Rabgap1l, Bdnf, Hnrnpk, Rspo3, C77370, Hnrnpu, Rybp, Ccnd1, Igf1, S/c16a3, Celsr3, Jub, Slc25a22, Clcn3, Kalrn, S/c38a3, Crebl2, Kank4, Slc7a2, Csf1, Kcnd3, Slc8a1, Cxcr4, Kcnip3, Smyd4, Dcp2, Klf4, Stc2, Dgkg, Magi2, Stk39, Dmpk, Mtap1a, Syn2, Elovl6, Mum1/1, Tcp11/1, Eml4, Mylk, Tgif2, Epb4.1/4b, Ngfr, Thrb, Fam101b, Nucks1, Zbtb4, Fbxw7, Pak3, Zfyve27 